\title{
Ein Interview mit Josef Richter
}

\author{
Josef Richter \\ vormals Wirtschaftskammer \\ Österreich
}

\author{
Johann Bacher \\ JKU Linz
}

\author{
Werner G. Müller \\ JKU Linz
}

\begin{abstract}
Das Interview mit Josef Richter wurde von Johann Bacher und Werner G. Müller am 28.3.2019 durchgeführt. Es beleuchtet die Entwicklungen in der amtlichen und angewandten Statistik insbesondere der Input-Output Analyse und Richters Rolle in der Österreichischen Statistischen Gesellschaft und bei der Entstehung des aktuellen Statistikgesetzes. Außerdem wird auf aktuelle Debatten eingegangen und ein Ausblick für das Fach Statistik gegeben.

Josef Richter war von 1967 bis 2003 in der Abteilung für Statistik in der Wirtschaftskammer tätig; 1979 bis 1990 auch Konsulent des Statistischen Zentralamts für methodische Fragen der Volkswirtschaftlichen Gesamtrechnung. Seit 1983 Lehrtätigkeit an der Universität Innsbruck und an der Universität Linz. Nach 2003 freiberuflich vor allem in Projekten für Eurostat tätig, darunter auch geblockte Vorlesungen für Mitarbeiter von Eurostat. Zahlreiche Forschungsarbeiten, vor allem auf dem Gebiet der Input-Output Analyse; mehr als 100 einschlägige Publikationen. Im Zentrum seines Interesses stehen derzeit vor allem Basiskonzepte der Statistik und die Möglichkeiten und Grenzen der empirischen Verankerung der Wirtschaftsforschung.
\end{abstract}

Keywords: interview, applied statistics, official statistics.

Werner Müller: Es ist für mich eine sehr große Freude, diese Reihe von Interviews, die wir für die Österreichische Statistische Zeitschrift begonnen haben, mit unserem Ehrenmitglied Dr. Josef Richter fort zu führen. Als Mitinterviewer habe ich den empirischen Sozialforscher, Professor für Soziologie an der JKU, Johann Bacher bei mir. Wir fangen am besten chronologisch an. Sie sind gebürtiger Wiener und haben auch in Wien das Studium bestritten. Damals gab es noch kein Studium der Statistik. Wie sind Sie also zu Ihrem Studium gekommen und woran können Sie sich besonders erinnern, was vielleicht auch schon in Beziehung zur Statistik gestanden ist?

Josef Richter: Ich habe an der Hochschule für Welthandel, wie sie damals hieß, jetzt Wirtschaftsuniversität, Volkswirtschaftslehre studiert und bin 1965 mit dem Diplom fertig geworden. Ich habe dann eine Dissertation geschrieben, bin aber nie zu den Rigorosen angetreten, weil ein Anschlag auf der Hochschule für Welthandel hing, Ökonometriker werden gesucht zur Erstellung eines mittelfristigen Input-Output-Modells. Wir haben alle nicht gewusst, was das ist, aber ein Freund, Werner Teufelsbauer, und ich haben gesagt, das klingt interessant, da bewerben wir uns. Dieses Modell war ein ambitiöses Projekt des Beirats für Wirtschafts- und Sozialfragen, denn damals war die ganz große Zeit der 
Sozialpartnerschaft. Nur um einige prominente Namen zu nennen: von der Gewerkschaft war Dr. Kienzl, von der Arbeiterkammer Dr. Staribacher und von der Wirtschaftskammer Dr. Klose tätig. Es war auch die Zeit der Planifikation, da hat man gemeint, wenn man ein solches Modell besäße, habe man fast alle wirtschaftspolitischen Probleme gelöst. Das Problem war nur, dass der Kern dieses Modells eine Input-Output-Tabelle sein sollte, und die gab es noch nicht. Und deshalb hat man die Arbeitsgruppe für mittelfristige Wirtschaftsprognosen geschaffen mit Experten der Arbeiterkammer, der Gewerkschaft, der Wirtschaftskammer. Ich bin dezidiert zu diesem Zweck in die Wirtschaftskammer (damals Bundeskammer der gewerblichen Wirtschaft) aufgenommen worden. Gerade zu dem Zeitpunkt, als der Chef des Statistischen Büros, Prof. Bruckmann, ausschied. Es dürfte eine seiner letzten Amtshandlungen gewesen sein, meinen Anstellungsvertrag zu unterschreiben. Bevor wir mit Ökonometrie oder Modellbau anfangen konnten, mussten wir einfach die Datengrundlagen schaffen. Das war sozusagen mein Einstieg in das Metier, wenn man so will. Wir haben damals die Input-Output-Tabelle 1964 kompiliert, und das war insofern etwas Besonderes, als es dann, als sie publiziert wurde, eine Gemeinschaftspublikation des Statistischen Zentralamtes, der Wirtschaftskammer und des Österreichischen Instituts für Wirtschaftsforschung war. Die Arbeitsteilung war so, dass das Statistische Zentralamt die Wertschöpfung, das war die Aufgabe von Dr. Franz, und einen Teil der Endverwendung erstellt hat, dafür war Mag. Schwarzl verantwortlich. Dr. Kramer, der nachmalige Chef des Wirtschaftsforschungsinstituts, hat die Investitionsspalten geschätzt. Den großen Intermediärteil haben Dr. Teufelsbauer, auch Wirtschaftskammer, Frau Dr. Geldner von der Arbeiterkammer und ich kompiliert. Das war gleichzeitig der Anfang der regelmäßigen „amtlichen“ Input-Output-Statistik in Österreich.

Werner Müller: Gut, das war dann schon an der Wirtschaftskammer angesiedelt, da waren Sie dann ja lange Zeit tätig (Anm. Richter: hauptberuflich eigentlich immer) und haben dann dort die Statistische Abteilung maßgeblich geprägt, waren dann ja auch Leiter der Statistischen Abteilung für längere Zeit. Welche anderen großen Vorhaben sind Ihnen noch wichtig und präsent?

Josef Richter: Man muss dazu sagen, dass man in dieser Zeit, damals hieß es ja noch Bundeskammer der gewerblichen Wirtschaft, ungemein wissenschaftsfreundlich war. Ich habe eigentlich perfekte Startbedingungen gehabt. Wenn ich irgendwohin zu einem Kongress hinfahren oder teure Bücher kaufen wollte, war es nie ein Problem. Die InputOutput-Tabelle 1964 ist im Frühjahr/Sommer 1972 fertig geworden. Da bestand die Arbeitsgruppe für mittelfristige Wirtschaftsprognosen des Beirats für Wirtschafts- und Sozialfragen schon nicht mehr; Dr. Teufelsbauer und ich haben dann das Modell in die Wirtschaftskammer übernommen. Wir konnten dann zur Erstellung des ersten Prototypen des Modells nach Amerika fahren, zu Prof. Clopper Almon an die University of Maryland in College Park. Prof. Almon habe ich überhaupt sehr, sehr viel zu verdanken. Das österreichische Modell war dann das erste der INFORUM Modellfamilie außer dem Modell für die Vereinigten Staaten. Man muss sich vorstellen, wir sind mit großen Schachteln mit Lochkarten gereist und auf der Rückreise sind uns peinlicherweise (es war alles in FORTRAN programmiert) die Lochkarten feucht geworden, die mussten einzeln dupliziert werden, das nur am Rande.

Eine weitere große Aufgabe ergab sich im Sommer 1972, gleich nach der Rückkehr aus den USA. Österreich hat am 01.01.1973 die Mehrwertsteuer eingeführt, es erfolgte also der Übergang von der Allphasenumsatzsteuer zur Mehrwertsteuer. Die große politische Frage war, wie viel Allphasenumsatzsteuer steckt in den einzelnen Preisen, also wie hoch müssen die vorgesehenen Entlastungssätze sein. Das ist eine Fragestellung, die man eigentlich nur mit Hilfe der Input-Output-Analyse lösen kann. Das war dann, bevor die Tabelle 1964 noch publiziert war, die erste Anwendung. Die errechneten Entlastungssätze sind, das passiert einem aber auch selten als empirischer Wirtschafts- 
forscher, in der Wiener Zeitung publiziert worden. Nur zur Illustration, es gab damals in Österreich eine einzige Maschine, mit der man eine $54 \times 54$ Matrix invertieren konnte, die stand am Rechenzentrum in Graz und ein Durchlauf hat so 6 - 7 Stunden gedauert, das war nur in der Nacht möglich. Also, ich kenne jetzt Graz aus dem Grund sehr gut.

Werner Müller: Sie haben ja überhaupt eine besondere Beziehung zu den Bundesländern, weil Sie dann früh auch begonnen haben, akademisch zu unterrichten, zuerst in Innsbruck und dann auch bei uns am Institut für sehr lange Zeit Lehrbeauftragter waren. Wie sind denn da Ihre Erinnerungen an die Innsbrucker Zeit?

Josef Richter: Ja, die Innsbrucker Zeit hat auch begonnen mit einem Input-Output-Projekt, mit etwas, das es vorher nie gab. Es wurde von Dr. Skolka vom Wirtschaftsforschungsinstitut angeregt, weil man das für viele Anwendungen der Input-Output-Analyse braucht, nämlich eine Tabelle zu den Preisen eines anderen Jahres, im Volksmund „Realtabelle“ genannt. Federführend dafür war in Innsbruck Prof. Hans-Werner Holub, der insofern bemerkenswert und für mich sehr bedeutend geworden ist, weil er eine Doppelprofessur hatte, für Volkswirtschaftslehre und für Statistik. Also ein extrem empirisch interessierter Volkswirt, leider eine große Seltenheit. Er war der offizielle Projektleiter, das Projekt wurde von der Nationalbank finanziert. Durchgeführt haben die Arbeiten Hofrat Schwarzl und ich. Da ist die Universität Innsbruck auf den Geschmack gekommen, solche Fragestellungen in die Lehre aufzunehmen. Ich habe ab dann zuerst relativ spezifische Vorlesungen zur Input-Output-Analyse gehalten. Später ging es mehr ins Allgemeine, in die empirische Wirtschaftsforschung und sehr stark in die empirischen Grundlagen der empirischen Wirtschaftsforschung. Das ist das Thema, das mich eigentlich immer am Meisten bewegt hat. Die Vorlesungen habe ich viele Jahre sehr, sehr gerne gemacht. Dann kam auch Linz dazu mit doch etwas anderen Schwerpunkten, wie Volkswirtschaftliche Gesamtrechnung und Wirtschaftsstatistik.

Werner Müller: Was dann in ihrem akademischen Lebenslauf auffällt ist, dass Sie Ihr Doktorat in Stuttgart nachgeholt haben. Wie ist das zustande gekommen?

Josef Richter: Ich hätte an der Hochschule für Welthandel schon eine approbierte Dissertation gehabt, habe mich aber nie aufraffen können, die letzten Prüfungen zu machen, weil ich schon so heftig im Modellbau und mit der Erstellung der Input-Output-Tabelle beschäftigt war. Prof. Holub hat vom ersten Moment an gesagt, ich muss mich habilitieren, da hab ich geantwortet, das geht wohl nicht, weil ich hab nicht mal ein Doktorat. Darauf hin hab ich dann in Stuttgart promoviert und zwar deswegen, weil es da einen Ordinarius, den Prof. Hermann Schnabl gab, der sehr stark in der Input-Output-Analyse spezialisiert war. Da war es irgendwo naheliegend, dort zu promovieren.

Werner Müller: Und folgerichtig gab's dann die Habilitation an der Universität Innsbruck für Volkswirtschaftslehre mit besonderer Berücksichtigung der empirischen Wirtschaftsforschung und der Wirtschaftsstatistik. Also da kommt die Wirtschaftsstatistik schon sehr prominent vor.

Josef Richter: Ja, man muss vielleicht überhaupt noch ergänzen, dass die Input-OutputTabelle 1964 auch für die amtliche Statistik ein Meilenstein war. Sie führte zu einer tiefgreifenden Revision der Volkswirtschaftlichen Gesamtrechnung, einer Revision, sehr eng verbunden mit Hofrat Dr. Franz.

Johann Bacher: Darf ich zur Input-Output-Analyse etwas fragen, die auch in der Soziologie in den 70-iger Jahren diskutiert wurde. Hatten Sie Kooperationen mit der Soziologie? Damals hat man versucht, z.B. die Zeitverwendung, also nicht volkswirtschaftliche Kennzahlen mit Input-Output-Tabellen zu erfassen.

Josef Richter: Ich habe mich neben dieser praktischen Tätigkeit, über die wir jetzt gesprochen haben, auch - quasi hobbymäßig - mit anderen, verwandten Dingen beschäftigt. Und 
wir haben schon 1971 mit Joachim Lamel und Werner Teufelsbauer für die große InputOutput Konferenz einen Beitrag über die Triangulierung von Input-Output-Tabellen gemacht. In solchen Tabellen sind die Wirtschaftsbereiche so anzuordnen, dass sie bestmöglich einer triangulierten Tabelle entsprechen, d.h. dass die Summe der Elemente über der Hauptdiagonale ein Maximum wird. Das hat in der Frühzeit eine Rolle gespielt, weil man schneller zu einer Lösung gekommen ist mit Gauß-Seidel. Aber wir haben das eigentlich inhaltlich, also ökonomisch gesehen: je weniger triangulär eine Tabelle ist, desto stärker ist die interne Verflechtung der Wirtschaft. Wir haben uns dann gesagt, den Ansatz müssten wir ja auch für andere Fragestellungen verwenden können und haben vor allem daran gedacht, die Konsistenz, die Transitivität von Wertesystemen, sowohl individuell als auch gesellschaftlich, zu erforschen. Ich denke, solche Systeme kann man nicht ganz analog zu Input-Output Tabellen triangulieren, da nur Ja/Nein Beziehungen bestehen und nicht quantifizierte Beziehungen. Wir haben damals auch mit einem Professor an der Universität Wien Kontakt aufgenommen, der ist aber nicht weiter darauf angesprungen. Das ist aber durchaus eine interessante Fragestellung.

Johann Bacher: Dieses Anwendungsgebiet der Input-Output-Analyse ist dann sozusagen eingeschlafen, während es in den 70-igern einige interessante Publikationen gegeben hat.

Josef Richter: Es gibt auch jetzt wieder solche Versuche, in denen man soziale Beziehungen, also Netzwerke, mit einem Ansatz der Input-Output-Analyse, der so genannten qualitativen Input-Output-Analyse, zu untersuchen versucht. Erst in der letzten Zeitschrift der Input-Output Association ist genau so ein Artikel erschienen.

Werner Müller: Kommen wir vielleicht zur Statistischen Gesellschaft. Sie sind ja schon sehr lange Mitglied der Statistischen Gesellschaft und sind dann leitend tätig gewesen, stellvertretender Präsident einmal und dann lange Zeit auch im Vorstand (Anm. Richter: über 20 Jahre). Was wollen Sie uns zur Statistischen Gesellschaft erzählen?

Josef Richter: Die Statistische Gesellschaft hat eine Gründungsidee, die mir sehr am Herzen liegt. Das Auseinanderentwickeln von akademischer Statistik, amtlicher Statistik und angewandter Statistik ein bisschen zu bremsen oder positiv ausgedrückt, die Kommunikation zwischen diesen Bereichen zu fördern. Das ist sozusagen das Hauptziel, wie ich es immer gesehen habe. Das zweite Ziel, und darum sind auch die Arbeitskreise gegründet worden, ist, ein Diskussionsforum zu schaffen, was besonders in der Sozialstatistik und in der Wirtschaftsstatistik sehr relevant ist. Ein Forum, in dem man unabhängig von den offiziellen Gremien, wo man natürlich Meinungen der Arbeiterkammer oder der Wirtschaftskammer usw. transportieren muss, frei von der Leber weg fachliche Fragen diskutieren kann. Das waren die eigentlichen Ziele.

Werner Müller: Gibt es irgendwelche besonderen Initiativen, die Sie in Erinnerung haben in ihrer Zeit in der ÖSG, die prägend waren?

Josef Richter: Die Gesellschaft hatte Aufschwünge und Abschwünge, was auch immer mit Personen verbunden war und ist. Da wurden z.B., als Dr. Franz Geschäftsführer war, sehr tolle Fachkonferenzen organisiert, einmal zur Regionalstatistik in Graz und - was jetzt wieder so modern geworden ist - zur ökologischen Gesamtrechnung in Baden bei Wien. Dann wurden sogar Tagungsbände herausgegeben.

Was mir in letzter Zeit sehr imponiert hat, war unter der Präsidentschaft von Grete Epler, dass man in der Veranstaltungsreihe „Politik und Statistik“ wirklich hochrangige Politiker eingeladen hat, zu Fragen der Statistik Stellung zu nehmen. Ich glaube, da hat man ein bisschen was bewirkt. Ich erinnere mich noch mit Freuden an den Vortrag und die Diskussion mit dem Abgeordneten zum Europaparlament Dr. Karas, der dann schon einige Male gesagt hat: „Ah so ist das. Können Sie mir dazu etwas Näheres sagen?" Oder an die Veranstaltungen mit z.B. Minister Hundsdorfer, Prof. Dr. Novotny und mit Dr. Wieser, das waren schon sehr nützliche Initiativen. Nicht zuletzt auch wegen 
des größer gewordenen Stellenwerts der Statistik in der Europäischen Union. Am Beginn meiner Statistikkarriere hat es diese eminente operationale Bedeutung statistischer Ergebnisse kaum gegeben. Die Volkszählungen waren zwar immer entscheidend für die Mandatsverteilung, relevant für den Finanzausgleich. Preisindizes sind in unzähligen Mietverträgen schon immer vorgekommen. Aber diese enorme politische Bedeutung, die die Statistik, insbesondere die Volkswirtschaftliche Gesamtrechnung, in der Europäischen Union bekommen hat, das hat's ja damals nicht gegeben. Mit all den Konsequenzen, vor allem, dass die amtliche Statistik extrem verrechtlicht worden ist; es ist eine völlig andere Welt geworden.

Werner Müller: Aber es gab natürlich auch Impulse von europäischer Seite zu Adaptierung, Novellierung oder Neugestaltung von gesetzlichen Vorgaben für die amtliche Statistik. War das auch der Impuls für das Statistikgesetz?

Josef Richter: Ja und Nein. Da gab es mehrere Faktoren. Der erste auslösende Faktor war der Betritt Österreichs zur Europäischen Gemeinschaft. Es gab ja vorher eine Volksabstimmung, die zu einer großen Mehrheit für den Beitritt geführt hat. Übrigens, das war sozusagen die schönste Anwendung des seit 1972 immer weiter ausgebauten InputOutput-Modells, mit dem ich einige Simulationen machen konnte, welche Wirtschaftsbereiche, welche Gütergruppen wie von so einem Betritt betroffen sein könnten.

Man hat vor der Abstimmung, ich habe intern immer wieder gesagt, das ist nicht gescheit, das sollte man nicht machen, sehr massiv argumentiert, dass der Beitritt zu gewaltigen bürokratischen Entlastungen führen wird: Das ganze Zollverfahren fällt weg, die Anmeldungen fallen weg, etc. Ja, und was dann kam, waren neue statistische Meldeverpflichtungen, vor allem zu "Intrastat“. Wir haben in den Jahren nach dem Beitritt eine echte Statistikakzeptanzkrise erlebt. Wenn einmal die Statistik auf den Titelseiten von Boulevardblättern steht, ist das nicht gut. Es ist genauso schlecht, wie wenn es einer Bank passiert. Es sind auf Hauptplätzen Fragebögen feierlich verbrannt worden, natürlich mit großem Medieninteresse. Und das war einer der Auslöser für ein neues Statistikgesetz.

Wir haben mit dem Beitritt das gesamte europäische statistische System übernehmen müssen. Das hat natürlich zu völligen Brüchen in vielen Zeitreihen geführt, auch mein schönes Input-Output-Modell ist daran gestorben. Denn wie schätzt man ökonometrisch Verhaltensgleichungen, wenn es keine Zeitreihen mehr gibt. Es war eine neue Welt. In Europa beruht ja ein Großteil der Statistik nicht auf Richtlinien, sondern auf Verordnungen, also unmittelbar rechtswirksamen Bestimmungen. Auch die Rolle der amtlichen Statistik hat sich damit sehr, sehr gewandelt. Vor 1995 hat man sich hingesetzt, wie es im Lehrbuch steht: Es lag eine analytische Fragestellung vor, und es ging darum, wie operationalisieren wir das, welche Konzepte verwenden wir, machen wir eine Erhebung oder was auch immer. Seitdem, und das ist ein Paradigmenwechsel, geht es primär um die Exekution von Verordnungen, deren Einhaltung kontrolliert wird. Der Paradigmenwechsel und die Statistikkrise waren sicher Auslöser für den Wunsch nach einem neuen Bundesstatistikgesetz.

Werner Müller: Wenn Sie sagen, das war einer der Auslöser, was gab es noch für andere Motive?

Josef Richter: Der weitere Auslöser war sicher eng verbunden mit dem neuen Datenschutzgesetz, auch vor dem Hintergrund der europäischen Vorgaben. Das waren umzusetzende Richtlinien, wenn ich mich richtig erinnere, und diese beiden Gesetze sind eigentlich parallel entstanden, auch ist derselbe Legist dafür zuständig gewesen. Der dritte Aspekt waren, wie gesagt, die massiven Beschwerden über die Respondentenbelastung und Budgetprobleme. Was damals sehr modern war und was sich auch im Bundesstatistikgesetz niedergeschlagen hat, war etwas, das sich New Public Management genannt hat. Dieser 
Ansatz führte zu der Lösung von Statistik Austria mit einem fachlichen und kaufmännischen Generaldirektor, das selbe findet man beim Tiergarten Schönbrunn und bei den Bundesmuseen usw., das war damals sozusagen „in“.

Johann Bacher: Vielleicht kommen wir nochmal zu der von Ihnen genannten Statistikkrise, der Respondentenbelastung, zurück. Von wem ist die ausgelöst worden, ist sie begründet entstanden oder medial inszeniert worden?

Josef Richter: Nein, nein. Die Firmen haben sich gefreut, sie brauchen keine Zollformulare mehr ausfüllen, wobei die Zollformulare üblicherweise der Spediteur ausgefüllt hat und nicht die Firma selbst. Jetzt kam aber die neue Verpflichtung, für alle Auslandstransaktionen, unabhängig ob import- oder ausfuhrseitig, sehr, sehr detailliert monatlich an die amtliche Statistik melden zu müssen. Wie dann später der Belastungsbarometer gezeigt hat, macht diese Erhebung Intrastat gut 50\% der gesamten Belastung in der Wirtschaft aus und das hat einen offenen Aufstand ausgelöst, mit parlamentarischen Anfragen, vielen Zeitungsartikeln.

Werner Müller: Dann gab`s auch für die Volkszählung so eine ähnliche Krise.

Josef Richter: Ja, in Deutschland viel intensiver.

Werner Müller: Vielleicht können Sie etwas skizzieren, wie Sie in die Rolle gekommen sind, bei der Neugestaltung um das Statistikgesetz mitzumachen.

Josef Richter: Wie ich wirklich dazugekommen bin, weiß ich nicht. Es war klar, der Gesetzesentwurf wird von Dr. Schittengruber, Ministerialrat im Bundeskanzleramt, dem Legisten, ausgearbeitet Er hat sich aber bei manchen Dingen ziemlich schwer getan und hat eines einschlägigen Rates bedurft. Wie das im Detail gelaufen ist, weiß ich nicht, jedenfalls wurden Dr. Kutzenberger und ich einbezogen. Das war insofern ganz nützlich, weil Ewald Kutzenberger mehr in der Demografie und der Sozialstatistik daheim ist und ich deutlich mehr wirtschaftsstatistische Schwerpunkte habe. Wir haben alle wirklich unheimlich viel dabei gelernt, als Staatsbürger, als Menschen. Diese Mitarbeit war eine wirklich prägende Erfahrung für mich, nicht zuletzt wegen des Erlebens der Kommunikationsprobleme/Sprachschwierigkeiten, die man mit einem Juristen hat.

Johann Bacher: War das eine Dreiergruppe oder eine größere Arbeitsgruppe, die am Gesetz gearbeitet haben?

Josef Richter: Es waren genau drei Personen.

Johann Bacher: Im Unterschied zu jetzt, wo die Reformgruppen deutlich größer sind (Anmerkung Müller: kleine Gruppen sind aber effektiver).

Josef Richter: Es gab sicher darüber hinaus Arbeitsgruppen, aber wir waren, wenn man es neumodisch sagt, die Task Force. Es gab eine ganze Reihe von politischen Vorgaben: Die erste war die Ausgliederung. Ausgliederung war damals modern: Tiergarten, Schloss Schönbrunn, Museen, Bundestheater, diese Institutionen waren vorher alle in der Hoheitsverwaltung, sie wurden damals alle ausgegliedert. Die zweite politische Vorgabe war, die Respondentenbelastung zu reduzieren. Drittens, es sind die neuen Datenschutzbestimmungen zu berücksichtigen. Ein goßes Schlagwort war damals die ,informationelle Selbstbestimmung". Gemeint war, wenn ich in diese eingreifen will, also in der Statistik Meldepflicht vorsehe, dann brauch ich dazu eine gesetzliche Grundlage. Und vielleicht die letzte politische Vorgabe, die ich erwähnen will, war die Notwendigkeit von massiven Einsparungen und der Personalreduktion in der amtlichen Statistik. Über die Doppelspitze und die Ausgliederung haben wir gar nicht reden müssen, das war vorher schon fixiert. 
Was Ewald Kutzenberger und ich beigetragen haben, und das war ursprünglich kein politisches Anliegen, waren einige Visionen einzubringen, wie eine amtliche Statistik auszusehen hat. Diese Anliegen haben wir auch nicht erfunden. Es gab damals schon die Fundamental Principles of Official Statistics der UNECE, die allerdings nicht rechtsverbindlich sind. Einige Elemente waren in der Europäischen Statistikverordnung aus dem Jahr 1997, im Artikel 10, niedergelegt, wo die Grundsätze der amtlichen Statistik formuliert werden. Aber worum es uns ging - und wo wir partiell erfolgreich waren - waren die folgenden Grundsätze einzubeziehen: Fachliche Autonomie bei der Erstellung von Statistik, Objektivität und eine völlig neue Publikationspolitik, die aus zwei Komponenten bestand. Einerseits gleicher, dem Umfang nach, und gleichzeitiger Zugang zu den Ergebnissen der Statistik („equal access“). Das war ganz arg umstritten, vielleicht können wir noch darüber sprechen. Und freier Zugang zu den statistischen Ergebnissen im Internet. Das haben wir im Statistikgesetz gegen großen Widerstand durchgebracht. Da haben wir eine echte Vorreiterrolle gespielt, zu dieser Zeit gab es von Eurostat wenige Daten frei im Internet verfügbar, Eurostat war da Jahre weit hinten.

Johann Bacher: Der Widerstand ist von der Statistik Austria gekommen?

Josef Richter: Ja, von daher auch. Wobei die Einnahmen aus dem Verkauf von Publikationen im Vergleich zum Budget wirklich nicht sehr bedeutend sind.

Im Gesetz wurde auch der Akzent auf die Verwendung von Verwaltungsdaten statt Erhebungsdaten gelenkt. Es hat die Statistik Austria ein Monopol für die Zuordnung der statistischen Einheiten bekommen. Es wundert mich noch heute, dass das durchgegangen ist. Es gab natürlich noch eine Menge anderer Anliegen, die wir nicht durchgebracht haben.

Johann Bacher: Können Sie uns dazu noch Beispiele nennen?

Josef Richter: Das Bundesstatistikgesetz bezieht sich nur auf die Bundesstatistik im engeren Sinn, daneben gibt es noch eine Menge anderer Statistiken, wie Ressortstatistiken. Unsere Vision war, zumindest eine gewisse Koordinierungskompetenz der Statistik Austria auf diesem Gebiet zu erreichen.

Werner Müller: Ein Ausfluss des Statistikgesetzes war auch der Statistikrat.

Josef Richter: Ein ganz wesentlicher Ausfluss, weil der Statistikrat hätte nach unserer Intention mehrere ganz wesentliche Aufgaben zu erfüllen gehabt, die er - offen gesagt leider nicht erfüllt hat. Wir haben uns erhofft, dass wir einige Aspekte, die wir im Gesetz nicht unterbringen konnten, wie zum Beispiel die Koordinierung über den engen Bereich der amtlichen Statistik hinaus, dass man das durch gute Gespräche im Statistikrat de facto erreichen kann. Darum war es so entscheidend, dass wichtige andere Statistikproduzenten, wie der Hauptverband der Sozialversicherungsträger, wie die Nationalbank, wie auch jene Ministerien, die Ressortstatistiken führen, dort vertreten sind.

Werner Müller: Woran ist das gescheitert, an Interessenskonflikten oder den handelnden Personen?

Josef Richter: In denke, primär an den Personen. Ich meine, die Statistik Austria hat das auch nicht sehr gefördert, dass muss man schon sagen. Ein weiteres Anliegen war die Qualitätssicherung, dazu gab es einen eigenen Ausschuss. Dort war der Statistikrat glaube ich - wirklich erfolgreich. Der erste Vorsitzende war Prof. Hackl, nachher war es Prof. Grossmann. Wir haben heute in Österreich für alle Statistikbereiche sogenannte Qualitätsberichte, die dem Nutzer sehr ausführliche Metadaten bereitstellen. Ich wage das zu sagen, weil ich viele solcher Berichte in anderen Ländern kenne, dass die österreichischen Qualitätsberichte vorbildlich sind. Der zweite Ausschuss war ein Ausschuss für das Arbeitsprogramm. Da war ich zehn Jahre lang der Vorsitzende, mit der Absicht, 
die Nutzeranliegen, die wir auch nicht adäquat ins Statistikgesetz hineingebracht haben, massiv zu vertreten. Es ging darum, dass die amtliche Statistik nicht nur das macht, was in europäischen Rechtsakten niedergelegt ist, sondern in einigen Fällen Dinge weiterführt, wo wir eine große Erfahrung und gute Tradition hatten, und wo es berechtige Anliegen der Forschung gibt. Ich muss offen sagen, da waren wir wenig erfolgreich. Wir haben uns sehr bemüht, in den Statistikrat Experten einzubeziehen, um dem Anliegen Gewicht zu verschaffen. Das war zuerst Dr. Kramer, Chef des Wirtschaftsforschungsinstituts, das war später von der Wirtschaftsuniversität Prof. Badelt.

Johann Bacher: Ich glaube, Sie sind ein bisschen zu pessimistisch, aus meiner Sicht...

Josef Richter: Ich fürchte, nein. Kurz gesagt, die Experten sind selten gekommen, sie haben kaum einmal in den Stellungnahmen zum Arbeitsprogramm ihre Interessen bekundet. Das ist eine traurige Erfahrung.

Johann Bacher: Ich kann mir vorstellen, dass das für Sie ein sehr mühsamer und teilweise frustrierender Prozess war. Trotzdem hat sich der Datenzugang, wenn ich das vergleiche zu meiner Zeit als Student oder dann später als wissenschaftlicher Mitarbeiter, verbessert. Man ist ja derzeit fast im Paradies: man kann den Mikrozensus anfordern, die EU-SILC-Daten. Bei den Zusatzerhebungen spießt es sich leider nach wie vor, weil die oft im Eigentum der Ministerien sind. Es gibt sicher noch Verbesserungsbedarf, aber vielleicht sind Sie ein wenig zu pessimistisch, was den Zugang betrifft.

Josef Richter: Was den reinen Datenzugang angeht, die Publikationen, die Daten, die kostenlos verfügbar sind, waren wir ein Vorreiter in Europa mit dem Statistikgesetz. Wo wir nicht erfolgreich waren, war die Interessen der Wissenschaft bei der Gestaltung des Arbeitsprogrammes durchzusetzen.

Werner Müller: Vielleicht kommen wir jetzt auf die aktuelle Debatte zu sprechen, weil die Umorganisation der Statistik Austria und dergleichen ist ja jetzt stark in den Medien. Und was auch immer wieder kolportiert wird, ist eine potenzielle Adaptierung, Veränderung des Statistikgesetzes. Wie sehen Sie da die Lage? Gibt es Gefahren der Verschlechterung oder sogar Möglichkeiten der Verbesserung?

Josef Richter: Ich muss gestehen, ich kenne die laufenden Diskussionen nicht genügend, vor allem kenne ich die Gesetzesentwürfe nicht. Ich bin nicht Mitglied dieser Arbeitsgruppe. Ich weiß im Prinzip nicht mehr, als was in den Zeitungen steht. Es gibt ein paar Problembereiche, die dürften jetzt wieder im Zentrum stehen, abgesehen von organisatorischen Themen. Wobei man sagen muss, die Doppelspitze ist ein ziemliches Unikat in Europa. Anzumerken ist dabei, dass es eine eigene Europäische Verordnung gibt zur Bestellung und Ablösung von Generaldirektoren. Was waren die großen Probleme beim Statistikgesetz? Das war, wie bereits gesagt, der Wunsch nach einer gewissen Autonomie beim Arbeitsprogramm. Wir haben damals mit Ewald Kutzenberger die Idee gehabt, man müsste dem fachlichen Generaldirektor so etwas wie eine „Privatschatulle“ für Projekte geben, die er für besonders wichtig hält, auch wenn es keine europäische Rechtsgrundlage gibt.

Werner Müller: Das ist offensichtlich nicht der Fall.

Josef Richter: ... Nein. Zusatzprogramme bedürfen der externen Finanzierung und damit des Auftrags eines Ministeriums. Das kann nicht jeder beauftragen, auch wenn sie einen privaten Financier finden, das ist nach dem Bundesstatistikgesetz nicht vorgesehen. Das ist ein großes Problem. Ich sehe die Gefahr des Rückzugs auf die Meldeverpflichtung gegenüber Europa. Ich kann keinen genauen Prozentsatz angeben, aber ich würde sagen, von der Tätigkeit von Statistik Austria entfallen mindestens 90 bis 95 Prozent auf Arbeiten zur Erfüllung einer europäischen Rechtsgrundlage. Es gibt sehr wenig anderes. 
Werner Müller: Was nun laufend kritisiert wird, dass nicht nur Datenbereitstellung, sondern auch Dateninterpretation usw. von der Statistik Austria durchgeführt wird. Wenn ich das recht verstehe, ist das im Statistikgesetz ja explizit so vorgesehen?

Josef Richter: Das war eine der ganz großen Diskussionen im Jahr 1999 mit einer gewissen Festlegung. Es gibt zwei Modelle unter den statistischen Ämtern. Das eine Modell ist, die statistischen Ämter produzieren die Daten, prüfen und evaluieren die Qualität der Daten und sehen, ob sie konsistent sind etc., machen aber keine weiterführenden Analysen. Das Gegenmodel ist das des französischen Amtes INSEE, aber auch des norwegischen Amtes; die haben große Forschungsabteilungen und erstellen in erheblichen Umfang Analysen. Damals ist die Entscheidung für das erste Modell gefallen. Ich war immer ein massiver Vertreter dieses Ansatzes, weil ich es demokratiepolitisch für äußerst problematisch halte, wenn am statistischen Amt eine Forschungsabteilung angedockt ist. Die hat naturgemäß einen solchen Informationsvorsprung, dass alle anderen Forschungseinrichtungen wenig Chancen haben.

Werner Müller: Das ist auch einer der Kritikpunkte der Opposition jetzt gerade: was Sie vorher erwähnt haben, diesen gleichen und gleichwertigen Zugang, dass der möglicherweise in Frage gestellt wird.

Josef Richter: Das war ein ganz, ganz großer Streitpunkt auch schon im Jahr 1999. Dieser Streit hat sich im Statistikrat immer wieder wiederholt. Es ist z.B. den Ressortvertretern, unabhängig von der politischen Coleur, schwierig zu vermitteln, dass die Daten, die sie finanzieren, nicht das „Privateigentum“ des Ministeriums sind. Ihre Sicht ist eine große Gefahr für den „equal access“, was den gleichen und gleichzeitigen Zugang zu Ergebnissen bedeutet. Das Prinzip des „equal access“ ist inzwischen in Europäischen Verordnungen festgelegt, das steht im Code of Practice, und das wird bei den Peer Reviews, die dazu dienen, die Umsetzung des Code of Practice in den einzelnen europäischen Ämtern zu kontrollieren, auch ganz intensiv überprüft.

Werner Müller: Weil Sie dies auch gerade ansprechen. Dieser Code of Practice, ist der nicht ohnehin Garantie dafür, dass sehr weitreichende Eingriffe in das Statistikgesetz kaum möglich sind, oder bin ich da naiv?

Josef Richter: Vom Gesetzestext her ja, von der Praxis her bin ich skeptisch. Ich war selbst in zwei Peer Reviews involviert. Ich habe feststellen können, dass die Gebräuche sehr verschieden sind, obwohl die Rechtslage eigentlich in der Verordnung völlig klar geregelt ist. Es gab z.B. Ämter, wo der Präsident des Amtes gleichzeitig Abteilungsleiter eines Ministeriums ist. Eine Situation, die eindeutig allen Grundsätzen widerspricht. Das wurde im Peer Review massiv kritisiert, und beim nächsten Peer Review wurde es wieder kritisiert, da es zwischenzeitlich keine Änderung gab.

Johann Bacher: Wenn Sie jetzt wieder eingeladen würden, in der Kommission mitzuarbeiten, was würden Sie an Reformvorschlägen einbringen?

Josef Richter: Meine zwei Hauptvorschläge wären sozusagen defensive Anliegen. Erstens, Wahrung des Prinzips des „equal access“. Das zweite zentrale Anliegen wäre die strikte Wahrung von was man salopp als „Einbahnprinzip“ bezeichnen könnte: Alle Verwaltungsdaten dürfen für statistische Zwecke verwendet werden (das steht auch so im Bundesstatistikgesetz), aber es dürfen statistische Daten nie zu Verwaltungszwecken herangezogen werden. Es gibt im Bereich der Statistik ein ausgezeichnetes Unternehmensregister, es gibt ein ausgezeichnetes Bevölkerungsregister. Die letzte Volkszählung wurde im Prinzip zum Großteil aus Verwaltungsdaten generiert. Ich glaube, da muss man sehr aufpassen, dass diese Informationen nicht für Verwaltungszwecke verwendet werden. Natürlich gibt es da Rationalisierungspotenziale, aber die Wahrung des Statistikgeheimnisses müsste Vorrang haben. 
Johann Bacher: Was könnte dazu beitragen, um das zu vermeiden? Die gesetzliche Bestimmung gibt es ja derzeit schon. Müssten da spezifische Organe, Institutionen geschaffen werden?

Josef Richter: Glaube ich eher nicht, das ist mehr eine Bewusstseinsfrage.

Werner Müller: Wie sehen Sie die potenzielle und ins Spiel gebrachte Unteraufsichtsstellung des Statistikamts unter das Parlament?

Josef Richter: Es gibt äußerst verschiedene Modelle in Europa, welche alle ihre Vor- und Nachteile haben. In manchen Ländern, wie in Deutschland, untersteht das Statistikamt, das nicht ausgegliedert ist, z.B. dem Innenministerium. Da finde ich unsere Regelung viel, viel besser. Wobei in Deutschland das damit zusammenhängt, dass das Statistikamt auch die Wahlbehörde ist. Schon jetzt gibt es einen Ansatz im Bundesstatistikgesetz, der nur noch keine besondere Bedeutung erlangt hat. Der Statistikrat hat jährlich die Aufgabe, einen Bericht über die Einhaltung der speziellen Grundsätze, zu denen der „equal access" gehört, aber auch das Verbot, politische Stellungnahmen abzugeben, etc... an den Bundeskanzler zu erstellen, der diesen dann dem Nationalrat zu übermitteln hat.

Werner Müller: Und, das ist noch nie passiert?

Josef Richter: Passiert ist es immer, aber dass es zu irgendwelchen Konsequenzen geführt hätte, kann ich mich nicht erinnern.

Johann Bacher: Für die Unabhängigkeit ist wichtig, eine fachlich kompetente, starke Leitung zu haben. Sehen Sie die Möglichkeit dies gesetzlich zu verankern? Derzeit steht ja genau nicht drinnen, was die fachliche Leitung für Kompetenzen haben muss. Sie kann ja relativ frei vom Kanzler ernannt werden. Würde es etwas bringen, wenn man die Vorgabe macht, dass das ein fachlich ausgebildeter Statistiker sein muss?

Josef Richter: Es gibt eine Europäische Verordnung, in der die genauen Vorgaben enthalten sind. Die ist natürlich rechtswirksam in Österreich.

Johann Bacher: Die Doppelspitze haben Sie angesprochen. Ihr Problem ist, dass die Stärke der Leitung geschwächt wird, da sich die Leitung gegenseitig blockieren kann.

Josef Richter: Die wirtschaftliche Leitung ist die stärkere nach dem österreichischen Bundesstatistikgesetz, das war uns sehr bewusst. Es wäre eine der Aufgaben des Statistikrates gewesen, z.B. im Interesse des Arbeitsprogramms, etwas dagegen zu steuern. Das ist nicht wirklich gelungen.

Johann Bacher: Sie sehen als wichtiges Organ den Statistikrat an. Müsste man da bei der Nominierung etwas ändern, damit der Statistikrat die Aufgaben, die Sie nennen, in einem besseren Ausmaß als derzeit erfüllen könnte?

Josef Richter: Wir haben uns damals sehr bemüht. Ich wüsste nicht, was man rein an den Bestimmungen ändern sollte. Eine der Bestimmungen ist z.B., dass zumindest ein Mitglied aus dem Kreis der vom Bundeskanzler zu Ernennenden ein Habilitierter sein muss. Das wirkliche Problem ist das nicht ausreichende Engagement mancher Mitglieder für die Anliegen des Statistikrates. Es ist ja nicht immer leicht, wenn man vom Ministerium dorthin entsandt ist. Im Gesetz steht zwar, dass das Mitglied zwar entsandt ist, aber völlig frei ist, sich zu emanzipieren von seiner Rolle als Interessensvertreter. Dass das so wenig gelungen ist, ist eigentlich eine der größten Frustrationen, die ich erlebt habe. Offen gesprochen, von den ursprünglich 15 Mitgliedern, haben sich, wenn es hochgegangen ist, vier bis fünf wirklich intensiv engagiert.

Johann Bacher: Die stärkere Einbeziehung der Wissenschaften der Universitäten, würde das etwas bringen? 
Josef Richter: Das war vorgesehen, teilweise auch sehr erfolgreich. Im Qualitätsausschuss, da ist wirklich etwas weitergegangen, zuerst durch Prof. Hackl und dann durch Prof. Grossmann. Die Nutzer haben sich leider oft nicht sehr eingebracht. Die hatten viele andere Möglichkeiten im Fachbereich, ihre Wünsche zu artikulieren. Ich bin mir bewusst, es ist mühsam und zeitaufwändig. Es gibt Sprachprobleme mit den Statistikern usw. Ich weiß nicht, wie es in der Soziologie ist, ich kann es nur in meinem Bereich sagen, in den Wirtschaftswissenschaften: Auch für die, die sich empirisch nennen, haben die empirischen Grundlagen ihrer Wissenschaft einen sehr geringen Stellenwert.

Johann Bacher: Ich glaube, hier bestehen Informationsdefizite auf Seiten der Wissenschaften. Mir z.B. war nicht bewusst, dass es überhaupt Möglichkeiten der Mitwirkung gibt. Es gibt ja keinen großen Call des Fachbereichs, „wir suchen Interessen oder Themenbereiche, die wir abdecken könnten. "Die Frage ist, wie und wo kann man als Wissenschaftler die Interessen artikulieren. Da fehlt vermutlich derzeit die Organisationsstruktur. Wie weiß jemand, der forscht, dass er bzw. sie thematische Interessen einbringen könnte?

Josef Richter: Das wäre auch eine Aufgabe der Statistischen Gesellschaft, das habe ich immer so gesehen.

Johann Bacher: Ein wichtiger Punkt ist das Vertrauen in die Statistik. Österreich hat ja keine besonders positive Einstellung zu Statistik und Zahlen. Man sieht das an der PisaDiskussion. Wie würden Sie aufgrund Ihrer Erfahrung das Vertrauen in die Statistik in Österreich einschätzen? Rückblickend - was waren Zeiten, wo besonderes hohes Vertrauen war, vielleicht die 1970er Jahre? Wann gab es geringeres Vertrauen? Sehen Sie die Gefahr, dass das Vertrauen zu gering wird, auch in Hinblick auf die zunehmende Verbreitung gefälschter Daten, so dass man sagt, das, was die Statistik Austria produziert, ist ohnedies alles erfunden?

Josef Richter: Ich glaube in der qualifizierten Öffentlichkeit ist das Vertrauen gegeben. Ich meine, wir haben in Österreich, das war schon auch eine Folge des Statistikgesetzes, in den letzten zwanzig Jahren einen gewaltigen Qualitätssprung gemacht; auch durch die Verpflichtung, verstärkt administrative Daten heranzuziehen. Die Statistik Austria hat heute eine Methodenabteilung, die sich wirklich sehen lassen kann, viele Absolventen aus diesem Hause sind dort tätig. Unter Leuten, die sich damit beschäftigen, ist die hohe Qualität anerkannt, auch wenn man immer etwas findet, was einem nicht optimal erscheint. Eine andere Geschichte ist die breite Öffentlichkeit. Eines der großen Defizite der Leitung der Statistik Austria war sicher, dass es nicht gelungen ist, das zu schaffen, was man in der Wirtschaft ein starkes Markenbewusstsein nennt. Wenn Sie sich die Zeitungen anschauen, dann finden Sie (Beispiele aus dem Wirtschaftsbereich) ganzseitige Berichte über Ergebnisse von irgendeinem Institut, von dem man noch nie etwas gehört hat. Ein Consultant führt eine Befragung von 60 Leuten durch, und das findet eine ganze Seite Resonanz in der Öffentlichkeit. Es gibt eine beachtliche Menge von Informationsmüll in den Zeitungen. Statistik Austria produziert zum Beispiel monatlich Konjunkturdaten unter höchsten Qualitätsansprüchen, kein Medium nimmt davon Kenntnis. Dr. Pesendorfer hat sich sehr bemüht, das zu ändern und er war partiell auch erfolgreich. Das geringe Bewusstsein der Öffentlichkeit um den besonderen Charakter der Ergebnisse der amtlichen Statistik ist ein ganz wesentliches Problem, das wird in Zukunft noch viel virulenter werden, wenn z.B. Google Analytics etwas publiziert. Wie grenze ich solche Aussagen gegenüber Resultaten der offiziellen Statistik ab? Google Analytics sind natürlich sehr schnell, im Vergleich zur amtlichen Statistik, die noch und noch Kontrollmechanismen einbauen muss. (Anmerkung Bacher: und argumentieren mit sehr großen Zahlen, eine Million, zwei Millionen oder mehr). Diese Konkurrenz wird ein echtes Problem für die amtliche Statistik werden.

Johann Bacher: Und die Einstellung der Politik und der Verwaltung, besteht Vertrauen in die Statistik? 
Josef Richter: Glaube ich schon in hohem Maße gegeben, ich wüsste auch nicht (Ergebnisse werden ja von Eurostat ziemlich genau kontrolliert), dass es da je irgendwelche gröberen Probleme gegeben hat, nicht so wie in Griechenland.

Werner Müller: Vielleicht können wir jetzt zurück kommen zu den Peer Review Tätigkeiten. Nachdem Sie dann aus der Wirtschaftskammer ausgeschieden sind, waren sie hier ja sehr aktiv und haben Peer Reviews durchgeführt?

Josef Richter: Je einen umfassenden Peer Review in Bulgarien und in Luxemburg, spezifische Reviews, die Wirtschaftsstatistik betreffend, in Georgien und Bosnien-Herzegowina.

Werner Müller: Und andere Aktivitäten auf europäischer Ebene? Sie sind ja nach wie vor sehr aktiv, in der Hinsicht. Was sind da die nächsten Pläne?

Josef Richter: Als ich 60 wurde, das ist schon lange her, wurde die Wirtschaftskammer wieder umorganisiert, man hat von vielen Mitarbeitern Abschied genommen, auch von mir, und man hat mich gleich wieder als Konsulent geholt. Ich habe den Freiraum genutzt, um verschiedenste wissenschaftliche Projekte zu machen, auch manches Exotische. Ich habe z.B. eine Input-Output-Tabelle für Brunei zusammen mit Kollegen vom Deutschen Institut für Wirtschaftsforschung erstellt, was auch ein Erlebnis besonderer Art war. Wenn Sie in einem Staat Volkswirtschaftliche Gesamtrechnungen betreiben, wo es keine Einkommensteuer, keine Sozialversicherung gibt, weil das alles vom Staat finanziert wird, der ja auf reichen Öl- oder Gasquellen sitzt, wo es keine Mehrwertsteuer gibt, dann fehlen Primärquellen, aus denen man üblicherweise schöpft.

Sonst habe ich hauptsächlich Projekte für Eurostat gemacht. Eine große Arbeit betraf die Inventur aller Rechtsbestände der Wirtschaftsstatistik. Mühsam, aber interessant, weil man so richtig dokumentieren konnte, wie inkonsistent dieses System ist, weil die einzelnen Verordnungen natürlich hintereinander, ohne einen Masterplan im Hintergrund, entstanden sind. Der damalige Präsident von Eurostat, Herr Radermacher, hat das vollkommen richtig erkannt, aber es ist unendlich schwierig, die Situation zu ändern. Eine meiner nächsten Aufgaben war es, eine solche Vision für Eurostat zu skizzieren, ein „Manual for business statistics“, eine Art „Blueprint“ für ein konsistentes wirtschaftsstatistisches System. Ich glaube nicht, dass das je umgesetzt wird. Das Bemühen um ein konsistentes System hat auch mit meinem Hintergrund eines Modellbauers zu tun, der erlebt, wie schrecklich es ist, wenn z.B. die Klassifikationen der einzelnen benötigten Statistiken nicht vergleichbar sind.

In den letzten Jahren haben Prof. Grossmann und ich für Eurostat an Ansätzen gearbeitet, die vielen Forschungsergebnisse im Bereich der amtlichen Statistik vielen Nutzern zugänglich zu machen. Auch oft problematisch und nicht immer erfolgreich, weil auch auf diesem Gebiet wenig koordiniert vorgegangen wird.

Werner Müller: Als Schluss ist das vom Topic her passend, vom Ton aber nicht. Es klingt sehr negativ, jetzt müssen wir irgendetwas Positives anhängen, vielleicht den Ausblick für unser Fach?

Josef Richter: Ich habe das Entwerfen von Konzepten und das empirische Arbeiten immer als extrem faszinierend empfunden, obwohl es stets mühsam ist. Aber der Ausgangspunkt ist die Neugierde, wie ist das eigentlich, wo gibt es Zusammenhänge?. (Anmerkung Müller: und das bleibt faszinierend auch für die nachfolgenden Generationen). Ja, und die empirische Arbeit sollte sich relevanten Themen widmen.

Werner Müller: Ich glaube das ist jetzt ein guter Schluss. Danke herzlichst für das Gespräch.

Die Interviewer bedanken sich herzlich bei Gabriele Mack-Niederleitner und Sandra ZeimlYudytskiy für die Transkription. 


\section{Affiliation:}

Josef Richter

vormals Wirtschaftskammer Österreich

und Universität Innsbruck

E-mail: josef.richter@aon.at

Johann Bacher

Department of Sociology

Johannes Kepler Universität Linz

A-4040 Linz, Austria

E-mail: johann.bacher@jku.at

URL: http://www.jku.at/institut-fuer-soziologie

Werner G. Müller

Department of Applied Statistics

Johannes Kepler Universität Linz

A-4040 Linz, Austria

E-mail: werner.mueller@jku.at

URL: http://www.jku.at/ifas

\section{Austrian Journal of Statistics}

published by the Austrian Society of Statistics

Volume 48

July 2019 http://www .ajs.or.at/

http://www.osg.or.at/

Accepted: 2019-05-10 